\title{
Chemical Kinetics and Inverse Modelling Problems
}

\author{
Victor Martinez-Luaces \\ Electrochemistry Engineering Multidisciplinary Research Group, \\ University of the Republic of Uruguay, Montevideo, \\ Uruguay
}

\section{Introduction}

Late last century and early this century, the Electrochemistry Engineering Multidisciplinary Research Group (Grupo Multidisciplinario de Ingenieria Electroquimica, in Spanish) at Montevideo, Uruguay studied the adsorption of Carbon Dioxide on Platinum surfaces (Zinola et al., 1997; Martinez-Luaces et al., 2001; Martinez-Luaces, 2001, Martinez-Luaces \& Guineo, 2002; Guineo \& Martinez-Luaces, 2002).

The research found three different adsorbates $E_{1}, E_{2}$, and $E_{3}$ and measured the surface concentrations by conventional electrochemical techniques.

The initial objective of the group was to propose a mechanism with the closest fit to the experimental curves (Zinola et al., 1997). Nevertheless, as the research progressed, the focus shifted to explain, or try to explain the double inflexion that consistently appeared in the experimental curves of surface concentration versus time for one of the adsorbates (Martinez-Luaces et al., 2001; Martinez-Luaces, 2001, Martinez-Luaces \& Guineo, 2002; Guineo \& Martinez-Luaces, 2002).

From a theoretical point of view, these are both inverse problems incorporating chemical and mathematical modelling at the same time. In previous papers and books, these problems were described as Inverse Modelling Problems (Martinez-Luaces, 2007, 2008, 2009a, 2009b, 2009c, 2009d).

In this paper, both inverse problems and the associated modelling are analysed from a theoretical point of view and subsequently solved. The main results will then be generalised to other Chemical Kinetics applications.

\section{Theoretical framework. Inverse problems}

Direct problems, according to Groestch (Groestch, 1999, 2001), can be regarded as those that provide the necessary information to follow a well-defined, stable procedure leading to a single correct solution.

Inverse problems, in contrast, are both more difficult and more interesting, largely due to their either having multiple solutions, or not being capable of being solved at all (Bunge, 
2006). They frequently crop up in the practice of several professions and careers. For instance, when treating a patient for a particular illness, listing the symptoms is a simple, direct problem that has already been solved and can be looked up in any medical textbook. On the other hand, diagnosing a patient's illness from his or her symptoms is not always a straightforward task, and requires an experienced doctor.

Inverse problems have not always been properly studied, and a quote from Bunge (Bunge, 2006) is appropriate: "The fact that almost all philosophers have ignored the peculiarities of inverse problems poses another inverse problem: to guess the reasons for this huge oversight on the part of philosophers."

In principle there are two different types of inverse problems, but in order to characterise them correctly, let us begin with a schematization of direct problems, adapting a study by Groetsch (Groestch, 1999, 2001). His scheme for a direct problem is like this:

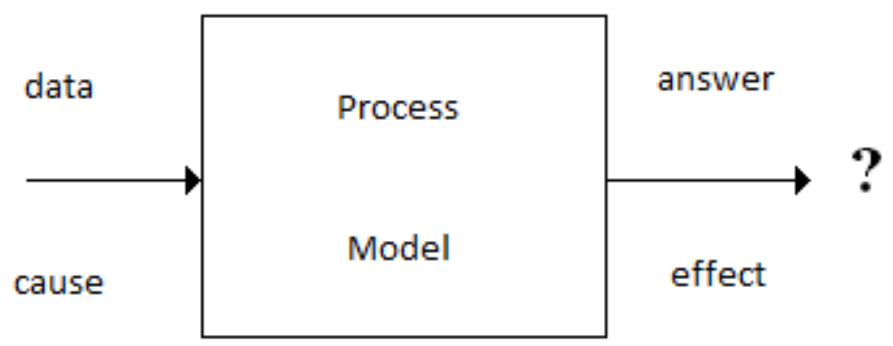

Fig. 1. Schema of direct problems

In the scheme in Figure 1, data and a given procedure are available, and the answer is sought; for instance, the reagents for a certain chemical reaction are given, and the conditions of temperature, $\mathrm{pH}$, etc of the reaction are known, and we wish to know the products of the reaction.

Now we can change the schema to obtain two inverse problems. The first is the causation problem, schematized in Figure 2:

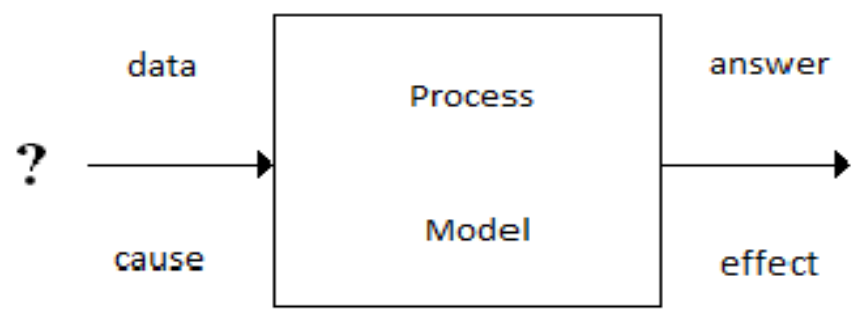

Fig. 2. Schema for causation problems

In this first type of inverse problem (causation problem), the results are known, as well as the process that produces them, but the causes are unknown. Finding the causes is the problem to be solved.

The other inverse problem generally encountered is the specification problem, schematized in Figure 3: 


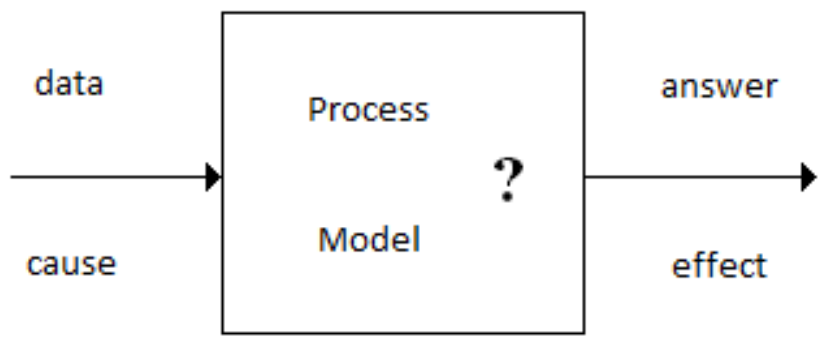

Fig. 3. Schema of specification problems

In this case, both the causes and the effects are known, and what must be determined is the process that leads from the former to the latter.

Both types of problems are common in the different branches of Chemistry. For example, in Qualitative Chemical Analysis one is frequently faced with a solution containing several unknown cations and anions. The analyst must carry out a pre-established series of reactions, and according to the results must rule out or confirm the presence or absence of the more usual cations and anions. This procedure allows the analyst, depending on the results (formation of precipitates, turbidity, colour reactions in the solution, etc.), to deduce the composition of the problem sample. Clearly, this is an example of a causation problem, because the procedure is pre-established, the results are in plain view and the objective is to know what solution composition is compatible with the results obtained (MartinezLuaces, 2011).

In Inorganic Chemistry, on the other hand, the problem is more frequently to synthesize a given salt from simpler substances (oxides, hydroxides, anhydrides, etc.). A typical example of this is the Solvay process for obtaining sodium carbonate $\left(\mathrm{Na}_{2} \mathrm{CO}_{3}\right)$ and sodium bicarbonate $\left(\mathrm{NaHCO}_{3}\right)$, used in the glass, paper and soap industries among others, from sodium chloride $(\mathrm{NaCl})$ and carbon dioxide $\left(\mathrm{CO}_{2}\right)$ which are much more abundant and easily obtained. The problem here does not lie in identifying the reactants and/or products, but in knowing and correctly carrying out the process that will lead to the formation of the desired products, starting from cheap and easily available reagents. This is clearly a specification problem (Martinez-Luaces, 2011).

Finally, in Organic Chemistry, double inverse problems (involving causation and specification) tend to be posed simultaneously. For instance, in organic synthesis, four different ways of preparing acetone are usually presented, starting from four different reagents: ethyl acetate, acetonitrile, acetaldehyde or 2-methylpropene. Obviously the reactants are not predetermined, much less the process, and only the final product or target molecule is known (Martinez-Luaces, 2011).

\section{Modelling and applications}

Problem solving, modelling and applications are not synonymous, although they are obviously related. For instance, the Discussion Document preparatory to the ICMI Study 14 (Blum et al., 2002), mentions that the term "modelling" focuses in the direction that goes from the real world towards basic sciences, while the term "applications" goes in the 
opposite direction, that is, from science towards the real world. In addition, the term "modelling" emphasizes the process that is taking place, while the word "applications" stresses the object involved (particularly in areas of the real world that are susceptible to a given mathematical treatment). The same document uses the term "problem" in a broad sense, including, therefore, not only practical problems, but also abstract problems, or those that attempt to describe, explain, understand, or even design parts of the real world.

Obviously, solving problems and modelling are not the same thing (one can model even in the absence of a concrete problem to be solved; one would simply be giving a mathematical description of a given phenomenon), and of course, "pure" mathematics problems, which do not require any kind of modelling, can be solved.

In the light of the above, we can arrive at the following schema:

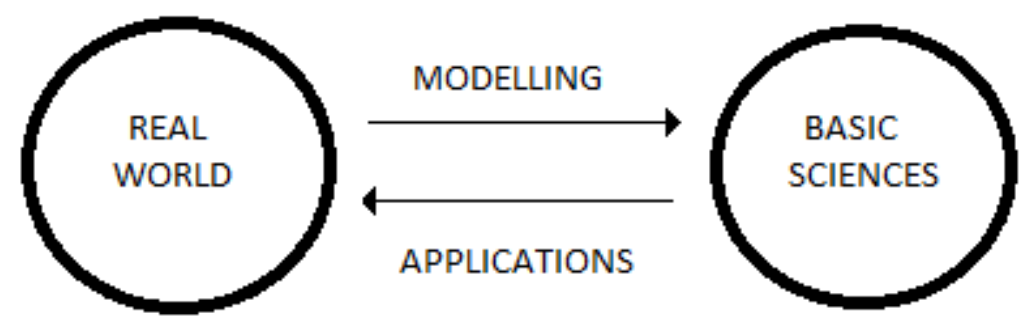

Fig. 4. Scheme of modelling and applications

Finally, it is worth mentioning that a more extensive discussion of modelling, applications and problem solving, and their teaching in university courses and secondary education in Latin America is available in previous papers (Martinez-Luaces, 2005, 2009a, 2011).

\section{The first problem. The mechanism proposal}

As it was mentioned before, the main objective of our research group was to study the adsorption of Carbon Dioxide on Platinum surfaces. The research found three different adsorbates $E_{1}, E_{2}$, and $E_{3}$ and measured the surface concentrations by conventional electrochemical techniques.

On completion of the trials of the experiment, several mechanisms were proposed and they were assessed by comparing the theoretical curves with the experimental ones. The result of this process was that the best fit was obtained by the following mechanism:

$$
\begin{aligned}
& \mathrm{E}_{1} \stackrel{k_{1}}{\longrightarrow} \mathrm{E}_{2} \\
& \mathrm{E}_{1} \stackrel{k_{2}}{\longrightarrow} \mathrm{E}_{3} \\
& \mathrm{E}_{2} \stackrel{k_{3}}{\longleftarrow} \mathrm{E}_{3}
\end{aligned}
$$

If $\left[E_{1}\right],\left[E_{2}\right]$ and $\left[E_{3}\right]$ represent the adsorbates' surface concentrations and $k_{1}, k_{2}, k_{3}$ and $k_{-3}$ the kinetic constants, then the mathematical model corresponding to this mechanism is the following: 


$$
\left\{\begin{array}{l}
\frac{d\left[E_{1}\right]}{d t}=-\left(k_{1}+k_{2}\right)\left[E_{1}\right] \\
\frac{d\left[E_{2}\right]}{d t}=k_{1}\left[E_{1}\right]-k_{3}\left[E_{2}\right]+k_{-3}\left[E_{3}\right] \\
\frac{d\left[E_{3}\right]}{d t}=k_{2}\left[E_{1}\right]+k_{3}\left[E_{2}\right]-k_{-3}\left[E_{3}\right]
\end{array}\right.
$$

or equivalently:

$$
\left(\begin{array}{l}
x \\
y \\
z
\end{array}\right)^{\prime}=\left(\begin{array}{ccc}
-k_{1}-k_{2} & 0 & 0 \\
k_{1} & -k_{3} & k_{-3} \\
k_{2} & k_{3} & -k_{-3}
\end{array}\right)\left(\begin{array}{l}
x \\
y \\
z
\end{array}\right)
$$

if notation is simplified.

It is interesting to observe that if the three rows of the Ordinary Differential Equations (ODE) system are added, the result is zero. This property remains true with other proposed mechanisms where only the solution will change. For example, consider the following mechanism:

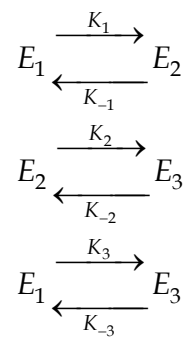

where all the possible reactions between the three adsorbates are considered.

For such a mechanism, the mathematical model (i.e., the Ordinary Differential Equations system) will be as follows:

$$
\left(\begin{array}{l}
x \\
y \\
z
\end{array}\right)^{\prime}=\left(\begin{array}{ccc}
-k_{1}-k_{3} & k_{-1} & k_{-3} \\
k_{1} & -k_{-1}-k_{2} & k_{-2} \\
k_{3} & k_{2} & -k_{-2}-k_{-3}
\end{array}\right)\left(\begin{array}{l}
x \\
y \\
z
\end{array}\right)
$$

As in the previous case, the surface concentrations $\left[E_{1}\right],\left[E_{2}\right]$ and $\left[E_{3}\right]$ were replaced by $x$, $y$ and $z$ in order to simplify notation.

Once again it is easy to observe that the result of adding the three rows of this new matrix is the null vector. This fact could be considered just a mathematical curiosity, though in the next section it will be the main observation to solve the second proposed problem.

\section{The second problem. The double inflexion}

The first proposed mechanism was the better when fitting the theoretical curves to the experimental values of surface concentration versus time. Nevertheless, one of the 
experimental curves consistently showed two inflexion points. Initially these inflexions were regarded as the uncertainty of the experimental measures, but each time the experiments were repeated, the results were always the same, i.e., one of the curves showed two inflexion points (Martinez-Luaces et al., 2001; Martinez-Luaces, 2001, Martinez-Luaces \& Guineo, 2002; Guineo \& Martinez-Luaces, 2002).

This led to other mechanisms being proposed, but none of them could explain this behaviour. As previously mentioned, all proposed mechanisms led to an O.D.E. system, for which the associated matrix had the property that the sum of all the matrix rows was zero. This observation is very useful because in all cases the matrix determinant must be zero. Finally, a null matrix determinant implies that one of the matrix eigenvalues is always zero and this determines the behaviour of the theoretical curves.

In order to explain these ideas, we shall consider a simpler chemical kinetics problem, with a simpler mechanism. For this purpose a well known process will be considered: the mutarotation of Glucose (Guerasimov, 1995; Martinez-Luaces, 2009e). In this mechanism there are only two simple uni-molecular reactions:

$$
\alpha \text {-Glucose } \stackrel{k_{1}}{\longleftarrow} \beta \text {-Glucose }
$$

This mechanism can be expressed mathematically through a simple ODE. In fact, if $x$ is the concentration of $\beta$-Glucose and $y$ that of $\alpha$-Glucose, then it is easy to observe that the $\beta$ Glucose concentration can be obtained from the following ODE:

$$
\frac{d x}{d t}=k_{1} \cdot y-k_{-1} x
$$

Letting $a$ be the initial $\alpha$ - Glucose concentration and the corresponding initial concentration of $\beta$ - Glucose being zero, then the variable $y$ can be replaced by $a-x$ and the ODE can be written as:

$$
\frac{d x}{d t}=k_{1} \cdot(a-x)-k_{-1} x
$$

Finally, very simple algebraic manipulations lead to the following linear, first order equation:

$$
\frac{d x}{d t}=k_{1} \cdot a-\left(k_{1}+k_{-1}\right) \cdot x
$$

The solution for this ODE is the following:

$$
x(t)=\frac{k_{1} a}{k_{1}+k_{-1}}\left\{1-\exp \left[-\left(k_{1}+k_{-1}\right) t\right]\right\}
$$

This is the typical approach for studying the Glucose Mutarotation problem (Guerasimov, 1995; Martinez-Luaces, 2009e), but not the best one for this article devoted to inverse problems. In order to get a different point of view, we shall write the mechanism: 


$$
\alpha \text {-Glucose } \stackrel{k_{1}}{\longleftarrow} \beta \text {-Glucose }
$$

in a simpler form as:

$$
A \stackrel{K}{\underset{\leftarrow}{\longrightarrow}} B
$$

In this case, a mathematical model, in terms of $\frac{d[A]}{d t} y \frac{d[B]}{d t}$ can be written in the following form:

$$
\left\{\begin{array}{l}
\frac{d[A]}{d t}=-K[A]+k[B] \\
\frac{d[B]}{d t}=K[A]-k[B]
\end{array}\right.
$$

Once again, if $x=[A]$ and $y=[B]$ the ODE system can be written in matrix form, as follows:

$$
\left(\begin{array}{l}
x \\
y
\end{array}\right)^{\prime}=\left(\begin{array}{cc}
-K & k \\
K & -k
\end{array}\right) \cdot\left(\begin{array}{l}
x \\
y
\end{array}\right)
$$

The system matrix is $M=\left(\begin{array}{cc}-K & k \\ K & -k\end{array}\right)$ and its eigenvalues can easily be obtained from the
characteristic equation:

$$
\operatorname{det}(M-\lambda I)=\operatorname{det}\left(\begin{array}{cc}
-K-\lambda & k \\
K & -k-\lambda
\end{array}\right)=0
$$

This process led to a second order equation $(-K-\lambda)(-k-\lambda)-K k=0$ that can be written as: $\lambda^{2}+(K+k) \lambda=0$ and the eigenvalues are: $\lambda_{1}=0$ and $\lambda_{2}=-(K+k)$. For these eigenvalues, the corresponding eigenvectors can be easily computed and the results are the following:

$$
\vec{v}_{1}=\left(\begin{array}{c}
k \\
K
\end{array}\right) \text { and } \vec{v}_{2}=\left(\begin{array}{c}
1 \\
-1
\end{array}\right) .
$$

In this case, the system matrix is equivalent to a diagonal form (Martinez-Luaces, 2009e) and the solution of the ODE problem is the following linear combination:

$$
\left(\begin{array}{l}
x(t) \\
y(t)
\end{array}\right)=\sum_{i} C_{i} \exp \left(\lambda_{i} t\right) \vec{v}_{i}=C_{1} \exp \left(\lambda_{1} t\right)\left(\begin{array}{l}
k \\
K
\end{array}\right)+C_{2} \exp \left(\lambda_{2} t\right)\left(\begin{array}{c}
1 \\
-1
\end{array}\right)
$$

and the final solution is:

$$
\left(\begin{array}{l}
x(t) \\
y(t)
\end{array}\right)=\left(\begin{array}{l}
C_{1} k+C_{2} e^{-(K+k) t} \\
C_{1} K-C_{2} e^{-(K+k) t}
\end{array}\right)
$$


More important than the solution itself - at least for this article - is to observe that one of the eigenvalues was zero (and the sum of both matrix rows was the null vector, as in the adsorption problem). This fact has important consequences for the asymptotic behaviour of the solutions. Moreover, as time approaches infinity:

$$
\lim _{t \rightarrow+\infty}\left(\begin{array}{l}
x(t) \\
y(t)
\end{array}\right)=\lim _{t \rightarrow+\infty}\left(\begin{array}{l}
C_{1} k+C_{2} e^{-(K+k) t} \\
C_{1} K-C_{2} e^{-(K+k) t}
\end{array}\right)=\left(\begin{array}{l}
C_{1} k \\
C_{1} K
\end{array}\right)
$$

The qualitative result is that both concentrations tend to finite, non-zero real values, which from a chemical point of view are the equilibrium concentrations.

Similar ideas can be explored when three or even more substances are involved. In order to explain this idea, we shall consider a very simple mechanism involving three different chemical substances $E_{1}, E_{2}$ and $E_{3}$, like the following one:

$$
E_{1} \stackrel{k_{1}}{\longrightarrow} E_{2} \stackrel{k_{2}}{\longrightarrow} E_{3}
$$

In this case, the ODE system (Martinez-Luaces, 2009e) is as follows:

$$
\left\{\begin{array}{l}
\frac{d\left[E_{1}\right]}{d t}=-k_{1}\left[E_{1}\right] \\
\frac{d\left[E_{2}\right]}{d t}=k_{1}\left[E_{1}\right]-k_{2}\left[E_{2}\right] \\
\frac{d\left[E_{3}\right]}{d t}=k_{2}\left[E_{2}\right]
\end{array}\right.
$$

or equivalently:

$$
\left(\begin{array}{l}
x \\
y \\
z
\end{array}\right)^{\prime}=\left(\begin{array}{ccc}
-k_{1} & 0 & 0 \\
k_{1} & -k_{2} & 0 \\
0 & k_{2} & 0
\end{array}\right)\left(\begin{array}{l}
x \\
y \\
z
\end{array}\right)
$$

where, as in the previous example, adding the rows the result is a null vector.

Applying the mass conservation law to the mechanism:

$$
E_{1} \stackrel{k_{1}}{\longrightarrow} E_{2} \stackrel{k_{2}}{\longrightarrow} E_{3}
$$

it follows that $\left[E_{1}\right]+\left[E_{2}\right]+\left[E_{3}\right]$ remains constant.

Finally, if $\left[E_{1}\right]+\left[E_{2}\right]+\left[E_{3}\right]=C$ is differentiated with respect to $t$, we obtain the following equation:

$$
\frac{d\left[E_{1}\right]}{d t}+\frac{d\left[E_{2}\right]}{d t}+\frac{d\left[E_{3}\right]}{d t}=0
$$


This formula explains why the sum of all the equations of the ODE system adds up to zero. From this observation, it follows that the sum of all the rows of the system matrix will always be the null vector (Martinez-Luaces, 2007, 2009e).

For the same reasons, all the possible mechanisms involving unimolecular reactions between all the adsorbates will show the same property. As a consequence of this fact, the system matrix has a null determinant for all the proposed mechanisms. This observation can be easily proved, because if $r o w_{1}+r o w_{2}+r o w_{3}=0$, then $r o w_{3}=-r o w_{1}-r o w_{2}$ and since each of the rows is a linear combination of the others, it follows that $\operatorname{det}(A)=0$, where $A$ is the matrix associated with the ODE system.

Therefore, if $\operatorname{det}(A)=0$, then matrix $A$ will have a null eigenvalue, which is independent of the mechanism proposed. For each mechanism the ODE system will have three eigenvalues: $\lambda_{1} \in R, \lambda_{2} \in R$ and $\lambda_{3}=0$. This observation has important consequences in the solutions of ODE systems and their qualitative behaviour. In order to get qualitative results, three different cases will be investigated:

1. all the eigenvalues are different,

2. two eigenvalues are the same and the other is a simple one

3. there exists an unique eigenvalue with algebraic multiplicity three.

All these cases will be analysed in order to discover whether the two inflexion points can be explained by mechanisms involving only electrochemical unimolecular reactions.

\subsection{First case: Three different eigenvalues $\left(\lambda_{1} \neq \lambda_{2}, \lambda_{1} \neq 0, \lambda_{2} \neq 0\right)$}

In this case, if $\vec{E}=\left(E_{1}, E_{2}, E_{3}\right)$, a well known formula - valid if all the eigenvalues are different - expresses that $\vec{E}=\sum_{i=1}^{3} c_{i} \exp \left(\lambda_{i} t\right) \vec{v}_{i}$, where $c_{i}$ represents the coefficients, $\lambda_{i}$ the eigenvalues and $\vec{v}_{i}$ the associated eigenvectors (Martinez-Luaces, 2009e). In fact, this formula was used to solve the mutarotation problem, as in (16). The same formula can be used for other diagonal forms with repeated eigenvalues.

Taking into account that $\lambda_{1} \neq 0, \lambda_{2} \neq 0$ and $\lambda_{3}=0$, all the surface concentrations have this form: $E_{i}=\alpha \exp \left(\lambda_{1} t\right)+\beta \exp \left(\lambda_{2} t\right)+\gamma$ and by differentiating we obtain: $\frac{d^{2} E_{i}}{d t^{2}}=\alpha \lambda_{1}^{2} \exp \left(\lambda_{1} t\right)+\beta \lambda_{2}^{2} \exp \left(\lambda_{2} t\right)=\exp \left(\lambda_{1} t\right)\left[\alpha \lambda_{1}^{2}+\beta \lambda_{2}^{2} \exp (\lambda t)\right]$ where $\lambda=\lambda_{2}-\lambda_{1}$, so $\frac{d^{2} E_{i}}{d t^{2}}=0$ if and only if $\alpha \lambda_{1}^{2}+\beta \lambda_{2}^{2} \exp (\lambda t)=0$, which only happens if $t=\frac{1}{\lambda} \ln \left(\frac{-\alpha \lambda_{1}^{2}}{\beta \lambda_{2}^{2}}\right)$. Then, in this case, only one inflexion point can be explained and so, two inflexion points are not possible.

\subsection{Second case: A double eigenvalue and a simple one}

There are three possibilities under this case: 


\subsubsection{First sub-case $\left(\lambda_{1} \neq 0\right.$ and $\left.\lambda_{2}=\lambda_{3}=0\right)$}

In this sub-case, the ODE system matrix $A$ is not equivalent to a diagonal matrix $D$, but it is equivalent to a triangular matrix $J$ (the Jordan canonical form). More precisely, there exists a matrix $P$ (it is important to note that in this case not all the columns of $P$ are eigenvectors) such that $P^{-1} A P=J$ and as a consequence, the ODE system $\dot{X}=A X$ can be easily converted in the following one $\dot{Y}=J Y$ by a simple change of variables $X=P Y$.

As a consequence of this fact, the new ODE system to solve is: $\left(\begin{array}{l}\dot{y}_{1} \\ \dot{y}_{2} \\ \dot{y}_{3}\end{array}\right)=\left(\begin{array}{lll}\lambda_{1} & 0 & 0 \\ 0 & 0 & 0 \\ 0 & 1 & 0\end{array}\right)\left(\begin{array}{l}y_{1} \\ y_{2} \\ y_{3}\end{array}\right)$ then $\dot{y}_{1}=\lambda_{1} y_{1}, \dot{y}_{2}=0$ and $\dot{y}_{3}=y_{2}$. The solution of this new ODE problem is $y_{1}=c_{1} \exp \left(\lambda_{1} t\right)$, $y_{2}=c_{2}$ and $y_{3}=c_{2} t+c_{3}$. Finally, since $X=P Y$, it follows that all the $E_{i}$ are linear combinations of these functions $y_{1}, y_{2}$ and $y_{3}$ and so, all of them are of the form: $E_{i}=\alpha \exp \left(\lambda_{1} t\right)+\beta t+\gamma$ and differentiating twice with respect to time, the result is: $\frac{d^{2} E_{i}}{d t^{2}}=\alpha \lambda_{1}^{2} \exp \left(\lambda_{1} t\right) \neq 0 \quad \forall t \in R$.

As a consequence of this fact, there are no inflexion points in this sub-case.

\subsubsection{Second sub-case $\left(\lambda_{1}=\lambda_{3}=0\right.$ and $\left.\lambda_{2} \neq 0\right)$}

This case is the same as the previous one, except for a change in the order of the eigenvalues, so, the main result is the same: there are no inflexion points in this sub-case.

5.2.3 Third sub-case $\left(\lambda_{1}=\lambda_{2} \neq 0\right.$ and $\left.\lambda_{3}=0\right)$

In this new sub-case, the corresponding Jordan canonical form has the following form:

$J=\left(\begin{array}{ccc}\lambda & 0 & 0 \\ 1 & \lambda & 0 \\ 0 & 0 & 0\end{array}\right)$, where $\lambda=\lambda_{1}=\lambda_{2}$ and the new ODE system is:

$\left(\begin{array}{l}\dot{y}_{1} \\ \dot{y}_{2} \\ \dot{y}_{3}\end{array}\right)=\left(\begin{array}{lll}\lambda & 0 & 0 \\ 1 & \lambda & 0 \\ 0 & 0 & 0\end{array}\right)\left(\begin{array}{l}y_{1} \\ y_{2} \\ y_{3}\end{array}\right)$ or equivalently $\dot{y}_{1}=\lambda y_{1}, \dot{y}_{2}=y_{1}+\lambda y_{2}$ and $\dot{y}_{3}=0$. Then, the solution of the first and the third ODE are $y_{1}=c_{1} \exp (\lambda t)$ and $y_{3}=c_{3}$. Finally, the second ODE can be written as $\dot{y}_{2}-\lambda y_{2}=c_{1} \exp (\lambda t)$ and the solution is $y_{2}=\left(c_{1} t+c_{2}\right) \exp (\lambda t)$.

Finally, taking into account the change of variables $X=P Y$, all the surface concentrations are of the following form: $E_{i}=(\alpha t+\beta) \exp (\lambda t)+\gamma$. Once again, if $E_{i}(t)$ is differentiated twice with respect to $t$, the result is: $\frac{d^{2} E_{i}}{d t^{2}}=\left(\lambda^{2} \alpha t+\lambda^{2} \beta+2 \lambda \alpha\right) \exp (\lambda t)$ As in previous cases, 
this expression can be zero only for a unique $t$ value, concretely $\frac{d^{2} E_{i}}{d t^{2}}$ is zero only for $t=\frac{-\lambda^{2} \beta-2 \lambda \alpha}{\lambda^{2} \alpha}$. Then, in this sub-case, only one inflexion point can be explained.

\subsection{Third case: A unique eigenvalue $\left(\lambda_{1}=\lambda_{2}=\lambda_{3}=0\right)$}

In this last case the Jordan canonical form is: $J=\left(\begin{array}{lll}0 & 0 & 0 \\ 1 & 0 & 0 \\ 0 & 1 & 0\end{array}\right)$ and the new ODE system is in this case: $\left(\begin{array}{l}\dot{y}_{1} \\ \dot{y}_{2} \\ \dot{y}_{3}\end{array}\right)=\left(\begin{array}{lll}0 & 0 & 0 \\ 1 & 0 & 0 \\ 0 & 1 & 0\end{array}\right)\left(\begin{array}{l}y_{1} \\ y_{2} \\ y_{3}\end{array}\right)$ or equivalently $\dot{y}_{1}=0, \dot{y}_{2}=y_{1}$ and $\dot{y}_{3}=y_{2}$. Then $y_{1}=c_{1}$, $y_{2}=c_{1} t+c_{2}$ and $y_{3}=c_{1} \frac{t^{2}}{2}+c_{2} t+c_{3}$ and taking into account the change of variables $X=P Y$, all the surface concentrations are second order polynomials, such as: $E_{i}=\alpha t^{2}+\beta t+\gamma$. Finally, double differentiation of $E_{i}(t)$ with respect to $t$, gives: $\frac{d^{2} E_{i}}{d t^{2}}=2 \alpha$ , so in this case there are no inflexion points.

As a consequence of this analysis, two inflexion points cannot be explained by mechanisms involving electrochemical uni-molecular reactions.

Finally, as mentioned in other papers, this double inflexion is explained in terms of electrocatalytic reactions (Martinez-Luaces, 2001: Guineo \& Martinez-Luaces, 2002).

\section{Other qualitative results}

As an example, let us consider again the following mechanism:

$$
E_{1} \stackrel{K_{1}}{\longleftarrow} E_{K_{-1}} \quad E_{2} \stackrel{K_{2}}{\longleftarrow} E_{3} \quad E_{1} \stackrel{K_{3}}{\longleftarrow} E_{3}
$$

where all the possible reactions between the three adsorbates are considered.

As it was mentioned before, for such a mechanism, the mathematical model is:

$$
\left(\begin{array}{c}
\dot{x} \\
\dot{y} \\
\dot{z}
\end{array}\right)=\left(\begin{array}{ccc}
-k_{1}-k_{3} & k_{-1} & k_{-3} \\
k_{1} & -k_{-1}-k_{2} & k_{-2} \\
k_{3} & k_{2} & -k_{-2}-k_{-3}
\end{array}\right)\left(\begin{array}{l}
x \\
y \\
z
\end{array}\right)
$$

In this problem - as in other models, corresponding to other mechanisms - the result of the sum of all the rows is the null vector, and this fact implies the existence of a null eigenvalue. 
In the previous section, this fact was utilised to prove the impossibility of two inflexion points in the surface concentration curves. Now, in this section, these facts will be used to reach other conclusions about qualitative behaviours, particularly in terms of stability of the solutions and several inverse problems related with this sequence of reactions.

\subsection{Stability of the solutions}

From a mathematical viewpoint when an ODE solution is altered slightly for instance, changing the initial conditions, a set of new curves may or may not show a different behaviour from the original one. From this fact, the heuristic basis for the concepts of stability and instability may be found. Moreover, a third possibility - asymptotically stable solutions - can arise when the altered solutions tend to the original one over a period of time, i.e., time nullifies any changes made to original solution (Martinez-Luaces, 2009e).

From a chemical point of view, the last option is the most desirable one, because it means that small errors due to measurements can be expected to diminish and almost disappear as reaction time proceeds.

In these cases, as in other mathematical models corresponding to ODE linear systems, stability and/or asymptotical stability strongly depend on the eigenvalues, particularly on their signs and multiplicity.

In all the unimolecular mechanisms studied there exists a null eigenvalue, and this fact implies that the solutions cannot be asymptotically stable (Martinez-Luaces, 2007, 2009e). As a consequence of chemical kinetics, small errors in the initial surface concentrations cannot be expected to vanish as the reaction tends to completion. Moreover, in the best hypothesis, we can expect these errors to be small and bounded, if the other eigenvalues are negative (i.e., this best possibility takes place only if $\lambda_{1}<0, \lambda_{2}<0$ and $\lambda_{3}=0$, or any other similar combination).

Let us return to the first mechanism considered in this article:

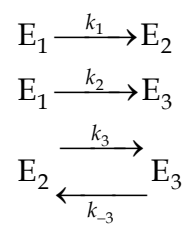

As mentioned before, the mathematical model corresponding to this mechanism is the following one:

$$
\left\{\begin{array}{l}
\frac{d\left[E_{1}\right]}{d t}=-\left(k_{1}+k_{2}\right)\left[E_{1}\right] \\
\frac{d\left[E_{2}\right]}{d t}=k_{1}\left[E_{1}\right]-k_{3}\left[E_{2}\right]+k_{-3}\left[E_{3}\right] \\
\frac{d\left[E_{3}\right]}{d t}=k_{2}\left[E_{1}\right]+k_{3}\left[E_{2}\right]-k_{-3}\left[E_{3}\right]
\end{array}\right.
$$


and the corresponding ODE system matrix is:

$$
A=\left(\begin{array}{ccc}
-k_{1}-k_{2} & 0 & 0 \\
k_{1} & -k_{3} & k_{-3} \\
k_{2} & k_{3} & -k_{-3}
\end{array}\right)
$$

In order to obtain the eigenvalues, the characteristic equation:

$$
\operatorname{det}(A-\lambda I)=\operatorname{det}\left(\begin{array}{ccc}
-k_{1}-k_{2}-\lambda & 0 & 0 \\
k_{1} & -k_{3}-\lambda & k_{-3} \\
k_{2} & k_{3} & -k_{-3}-\lambda
\end{array}\right)=0
$$

must be solved.

Algebraic manipulations lead to the following equation:

$-\lambda\left(\lambda+k_{1}+k_{2}\right)\left(\lambda+k_{3}+k_{-3}\right)=0$ and the solutions are $\lambda_{1}=0, \lambda_{2}=-k_{1}-k_{2}<0$ and $\lambda_{3}=-k_{3}-k_{-3}<0$. Then, in this case two eigenvalues are negative and the other one is zero and so, solutions of the ODE system are stable, but not asymptotically.

Let us turn again to the simpler mechanism, already studied:

$$
E_{1} \stackrel{k_{1}}{\longrightarrow} E_{2} \stackrel{k_{2}}{\longrightarrow} E_{3}
$$

In this case, the ODE system is as follows:

$$
\left\{\begin{array}{l}
\frac{d\left[E_{1}\right]}{d t}=-k_{1}\left[E_{1}\right] \\
\frac{d\left[E_{2}\right]}{d t}=k_{1}\left[E_{1}\right]-k_{2}\left[E_{2}\right] \\
\frac{d\left[E_{3}\right]}{d t}=k_{2}\left[E_{2}\right]
\end{array}\right.
$$

and the matrix system is:

$$
A=\left(\begin{array}{ccc}
-k_{1} & 0 & 0 \\
k_{1} & -k_{2} & 0 \\
0 & k_{2} & 0
\end{array}\right)
$$

In this simpler case it is not necessary to solve the characteristic equation, i.e. $\operatorname{det}(A-\lambda I)=0$, because $A$ is a lower triangular matrix and so, the eigenvalues are the diagonal elements: $\lambda_{1}=-k_{1}<0, \lambda_{2}=-k_{2}<0$ and $\lambda_{3}=0$. Again, in this case, solutions are stable, but not asymptotically, and so, experimental errors in the initial conditions tend to remain small and bounded, but they will not tend to disappear with time.

As a final example, let us consider again the following mechanism involving all the possible reactions between the three adsorbates: 


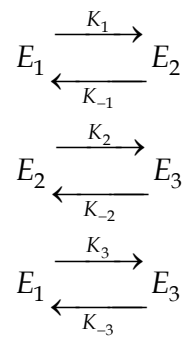

The mathematical model for this mechanism, written in a simplified vector form is:

$$
\left(\begin{array}{l}
x \\
y \\
z
\end{array}\right)^{\prime}=\left(\begin{array}{ccc}
-k_{1}-k_{3} & k_{-1} & k_{-3} \\
k_{1} & -k_{-1}-k_{2} & k_{-2} \\
k_{3} & k_{2} & -k_{-2}-k_{-3}
\end{array}\right)\left(\begin{array}{l}
x \\
y \\
z
\end{array}\right)
$$

And the characteristic equation is:

$$
\operatorname{det}(A-\lambda I)=\operatorname{det}\left(\begin{array}{ccc}
-k_{1}-k_{3}-\lambda & k_{-1} & k_{-3} \\
k_{1} & -k_{-1}-k_{2}-\lambda & k_{-2} \\
k_{3} & k_{2} & -k_{-2}-k_{-3}-\lambda
\end{array}\right)=0
$$

Once again, algebraic manipulations lead to a third order polynomial equation of this form: $-\lambda^{3}-a \lambda^{2}-b \lambda=0$ that can be written as $(-\lambda)\left(\lambda^{2}+a \lambda+b\right)=0 \quad$ where $a=k_{1}+k_{2}+k_{3}+k_{-1}+k_{-2}+k_{-3}=\sum_{i} k_{i}>0$ and $b=k_{1} k_{2}+k_{1} k_{-2}+k_{1} k_{-3}+k_{2} k_{3}+k_{2} k_{-3}+k_{3} k_{-1}+k_{3} k_{-2}+k_{-1} k_{-2}+k_{-1} k_{-3}>0$.

On the other hand, since the eigenvalues are $\lambda_{1}, \lambda_{2}$ and $\lambda_{3}=0$, this polynomial equation can be expressed as: $\left(\lambda_{1}-\lambda\right)\left(\lambda_{2}-\lambda\right)(0-\lambda)=0$ which can be written in the alternative form: $(-\lambda)\left(\lambda^{2}-\left(\lambda_{1}+\lambda_{2}\right) \lambda+\lambda_{1} \lambda_{2}\right)=0$.

Finally, if $(-\lambda)\left(\lambda^{2}+a \lambda+b\right)=0$ and $(-\lambda)\left(\lambda^{2}-\left(\lambda_{1}+\lambda_{2}\right) \lambda+\lambda_{1} \lambda_{2}\right)=0$ are different forms of the same equation, then: $\lambda_{1}+\lambda_{2}=-a<0$ and $\lambda_{1} \lambda_{2}=b>0$. These inequalities lead to the following result: $\lambda_{1}<0, \lambda_{2}<0$ and $\lambda_{3}=0$, as in the other examples previously analysed and, once again, this fact means that the ODE solutions will be stable, but not asymptotically.

In all the previous examples, results can be summarised as: $\lambda_{1}<0, \lambda_{2}<0$ and $\lambda_{3}=0$ or any other similar combination. It is important to mention that the null eigenvalue can be obtained simply as a consequence of Lavoisier's law, as shown previously (in section 5), while the others must be obtained from the characteristic equation.

In all cases analysed solutions are just stable (a weak stability, not asymptotic stability) and as a consequence, experimental errors in the initial concentrations tend to remain bounded, but they do not tend to vanish with time.

\subsection{Inverse modelling problems}

Each chemical or electrochemical mechanism involving unimolecular reactions leads to a linear ODE system. This is a well known result, but what happens with the inverse question: 
does any ODE linear system correspond - at least theoretically - to a given mechanism? If not, what condition must be considered to ensure that a given ODE linear system corresponds to a chemical unimolecular reaction mechanism?

In order to answer these questions we shall slightly modify the ODE system matrix corresponding to one of the mechanisms previously analysed. For instance, if we consider again the following mechanism:

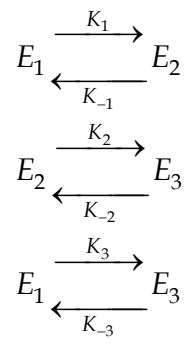

and the corresponding ODE system matrix is:

$$
A=\left(\begin{array}{ccc}
-k_{1}-k_{3} & k_{-1} & k_{-3} \\
k_{1} & -k_{-1}-k_{2} & k_{-2} \\
k_{3} & k_{2} & -k_{-2}-k_{-3}
\end{array}\right)
$$

Now, if just the first entry of this system matrix is modified, the resulting matrix will be:

$$
A_{\varepsilon}=\left(\begin{array}{ccc}
\varepsilon-k_{1}-k_{3} & k_{-1} & k_{-3} \\
k_{1} & -k_{-1}-k_{2} & k_{-2} \\
k_{3} & k_{2} & -k_{-2}-k_{-3}
\end{array}\right)
$$

(it is important to note that the first entry $a_{11}=-k_{1}-k_{3}$ was changed to a slightly different new one, $\left.a_{11}^{*}=-k_{1}-k_{3}+\varepsilon\right)$.

This new matrix $A_{\varepsilon}$ does not correspond to a certain reaction mechanism, because if all its rows are added, the result is: $(\varepsilon, 0,0)$ instead of the null vector as in the case of matrix A.

This observation has a dramatic consequence for the inverse modelling problem: $A$ corresponds to a given unimolecular reaction mechanism, but $A_{\varepsilon}$ cannot be associated with any of these mechanisms. As mentioned before, this is due to the mass conservation law, which in this case can be written as $\left[E_{1}\right]+\left[E_{2}\right]+\left[E_{3}\right]=c$. Derivation of this equation gives the following: $\frac{d[A]}{d t}+\frac{d[B]}{d t}+\frac{d[C]}{d t}=0$ and the last formula implies that adding all the matrix rows the result must be zero. This criterion can be used in order to know 
whether or not a given matrix corresponds to a certain mechanism (Martinez-Luaces, 2007, 2009a, 2009e).

\section{Conclusions and observations}

This article describes a combination of chemical and mathematical modelling applied to the adsorption of Carbon Dioxide on Platinum surfaces, but a similar procedure can be applied to any chemical or electrochemical mechanism involving unimolecular reactions. Moreover, mathematical theorems about eigenvalues, eigenvectors, diagonalization, Jordan canonical forms, etc., and chemical laws, particularly Lavoisier's law of mass conservation can be combined to solve inverse causation and stability problems.

It is well known that any given chemical mechanism involving unimolecular reactions leads to a linear ODE system, but the converse is not true. In fact, as previously shown, slightly modifying a given ODE system matrix results in a new matrix that does not correspond to any unimolecular reaction mechanism. Then, this result gives a negative answer to the inverse problem about whether a mechanism exists for every linear ODE system. At the same time, it gives a negative answer to the "inverse stability problem", i.e., a slight change in one of the coefficients produces dramatic changes in the inverse problem. Moreover, there exists a mechanism corresponding to a certain matrix $A$ but not for the modified matrix $A_{\varepsilon}$. This is a typical conclusion of inverse problems, where existence, uniqueness and stability do not usually occur.

Finally, other issues like the qualitative behaviour of solutions (stability, instability and asymptotic stability), can be analysed using this approach. Moreover, in all the examples studied, solutions exhibited weak stability, which in terms of the electrochemical problem means that experimental errors in the initial surface concentrations tend to remain limited as the reaction proceeds to completion. Nevertheless, these experimental errors never tend to vanish, and this fact is a consequence of having a null eigenvalue. As observed earlier, all these facts can be proved combining mathematical theorems with chemical laws, so they can be easily generalised to other unimolecular reactions mechanisms.

\section{References}

Blum, W. et al., (2002). ICMI Study 14: applications and modelling in mathematics education - discussion document. Educational Studies in Mathematics, Vol. 51, No. 1-2, 149171.

Bunge, M., (2006), Problemas directos e inversos, In: Grupobunge. Filosofia y ciencia, Access: May, 2011, Available from:

http://grupobunge.wordpress.com/2006/07/20/119.

Groestch, C.W., (1999), Inverse problems: activities for undergraduates, Mathematical Association of America (Eds.), Washington D.C., U.S.A.

Groestch, C.W., (2001). Inverse problems: the other two-thirds of the story, Quaestiones Mathematicae, Vol. Suppl. 1, 2001, Supplement, 89-93, ISSN 1607-3606.

Guerasimov, Y.A., et al., (1995), Physical Chemistry, 2nd Edition, Houghton Mifflin, (Eds.) Boston, U.S.A. 
Guineo Cobs, G. \& Martínez Luaces, V., (2002). Electrocatalytic reactions: An interesting problem of Numerical Calculus, Communications of the $2^{\text {nd }}$ International Conference on the Teaching of Mathematics at the undergraduate level. Crete, Greece, July 2002.

Martínez Luaces, V., (2001). Reacciones Electroquímicas y Electrocatalíticas: Un problema de Matemática Aplicada. Actas COMAT 95-97-99, ISBN 959 - 160097 - 6. Matanzas, Cuba.

Martínez Luaces, V., (2005). Engaging Secondary School and University Teachers in Modelling: Some Experiences in South American Countries. International Journal of Mathematical Education in Science and Technology, Vol 36, No. 2-3, pp. 193-194, ISSN 0020-739X.

Martínez Luaces, V., (2007). Inverse-modelling problems in Chemical Engineering courses. Vision and change for a new century. Proceedings of Calafate Delta '07. ISBN: 978-997496-348-1, El Calafate, Argentina, November 2007.

Martínez Luaces, V., (2008). Modelado inverso y Transformada de Laplace en problemas de diseño de reactores químicos. Proceedings of EMCI XIV. Educación Matemática en Carreras de Ingeniería, Mendoza, Argentina, May 2008.

Martínez Luaces, V., (2009a). Modelling and inverse-modelling: experiences with O.D.E. linear systems in engineering courses, International Journal of Mathematical Education in Science and Technology, Vol. 40, No. 2, pp. 259-268, ISSN 0020-739X.

Martínez Luaces,V., (2009b). Problemas de modelado directo e inverso con Ecuaciones Diferenciales y Transformación de Laplace. Proceedings of EMCI XV. Educación Matemática en Carreras de Ingeniería, Tucumán, Argentina, September 2009.

Martínez Luaces,V., (2009c). Modelling and Inverse Modelling with second order P.D.E. in Engineering courses. Proceedings of Southern Right Delta '09. Gordon's Bay, South Africa, November 2009.

Martínez Luaces,V., (2009d). Modelling, applications and Inverse Modelling: Innovations in Differential Equations courses. Proceedings of Southern Right Gordon's Bay Delta '09, Gordon's Bay, South Africa, November 2009.

Martínez Luaces, V., (2009e). Aplicaciones y modelado: Ecuaciones Diferenciales Ordinarias, Transformación de Laplace, Ecuaciones en Derivadas Parciales. Matser (Eds.), ISBN 9789974-96-621-5 Montevideo, Uruguay.

Martínez Luaces, V., (2011). Problemas inversos: los casi olvidados de la Matemática Educativa. Acta Latinoamericana de Matematica Educativa, 24, pp. 439-447. ISBN 978607-95306-4-8.

Martínez Luaces, V. \& Guineo Cobs, G., (2002). Un problema de Electroquímica y su Modelación Matemática, Anuario Latinoamericano de Educación Química. Año 2002, pp. 272 - 276, ISSN 0328 - 087X.

Martínez Luaces, V., Zinola, F. \& Méndez, E., (2001). Problemas Matemáticos Computacionales en el estudio de Mecanismos de Reacciones Químicas", Actas de COMAT 95-97-99, ISBN 959 - 160097 - 6. Cuba. 
Zinola, F., Méndez, E. \& Martínez Luaces, V., (1997). Modificación de estados adsorbidos de Anhídrido Carbónico reducido por labilización electroquímica en superficies facetadas de platino. Proceedings of X Congreso Argentino de Fisicoquímica, Tucumán, Argentina, April 1997. 


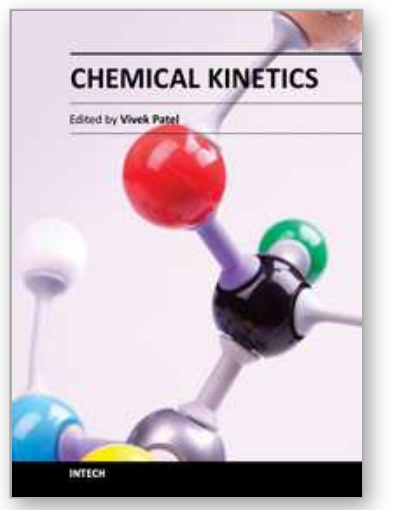

\author{
Chemical Kinetics \\ Edited by Dr Vivek Patel
}

ISBN 978-953-51-0132-1

Hard cover, 344 pages

Publisher InTech

Published online 29, February, 2012

Published in print edition February, 2012

Chemical Kinetics relates to the rates of chemical reactions and factors such as concentration and temperature, which affects the rates of chemical reactions. Such studies are important in providing essential evidence as to the mechanisms of chemical processes. The book is designed to help the reader, particularly students and researchers of physical science, understand the chemical kinetics mechanics and chemical reactions. The selection of topics addressed and the examples, tables and graphs used to illustrate them are governed, to a large extent, by the fact that this book is aimed primarily at physical science (mainly chemistry) technologists. Undoubtedly, this book contains "must read" materials for students, engineers, and researchers working in the chemistry and chemical kinetics area. This book provides valuable insight into the mechanisms and chemical reactions. It is written in concise, self-explanatory and informative manner by a world class scientists in the field.

\title{
How to reference
}

In order to correctly reference this scholarly work, feel free to copy and paste the following:

Victor Martinez-Luaces (2012). Chemical Kinetics and Inverse Modelling Problems, Chemical Kinetics, Dr Vivek Patel (Ed.), ISBN: 978-953-51-0132-1, InTech, Available from:

http://www.intechopen.com/books/chemical-kinetics/chemical-kinetics-and-inverse-modelling-problems

\section{INTECH}

open science | open minds

\author{
InTech Europe \\ University Campus STeP Ri \\ Slavka Krautzeka 83/A \\ 51000 Rijeka, Croatia \\ Phone: +385 (51) 770447 \\ Fax: +385 (51) 686166 \\ www.intechopen.com
}

\author{
InTech China \\ Unit 405, Office Block, Hotel Equatorial Shanghai \\ No.65, Yan An Road (West), Shanghai, 200040, China \\ 中国上海市延安西路65号上海国际贵都大饭店办公楼405单元 \\ Phone: +86-21-62489820 \\ Fax: $+86-21-62489821$
}


(C) 2012 The Author(s). Licensee IntechOpen. This is an open access article distributed under the terms of the Creative Commons Attribution 3.0 License, which permits unrestricted use, distribution, and reproduction in any medium, provided the original work is properly cited. 